\title{
Viscous Force Retards Initial Droplets Spreading
}

\author{
Lichun Shi, Yang Liu, Hongyu Lu, Yonggang Meng, Guoqing Hu, and Yu Tian
}

J. Phys. Chem. C, Just Accepted Manuscript • DOI: 10.1021/acs.jpcc.7b06124 • Publication Date (Web): 19 Sep 2017

Downloaded from http://pubs.acs.org on September 20, 2017

\section{Just Accepted}

"Just Accepted" manuscripts have been peer-reviewed and accepted for publication. They are posted online prior to technical editing, formatting for publication and author proofing. The American Chemical Society provides "Just Accepted" as a free service to the research community to expedite the dissemination of scientific material as soon as possible after acceptance. "Just Accepted" manuscripts appear in full in PDF format accompanied by an HTML abstract. "Just Accepted" manuscripts have been fully peer reviewed, but should not be considered the official version of record. They are accessible to all readers and citable by the Digital Object Identifier (DO|®). "Just Accepted" is an optional service offered to authors. Therefore, the "Just Accepted" Web site may not include all articles that will be published in the journal. After a manuscript is technically edited and formatted, it will be removed from the "Just Accepted" Web site and published as an ASAP article. Note that technical editing may introduce minor changes to the manuscript text and/or graphics which could affect content, and all legal disclaimers and ethical guidelines that apply to the journal pertain. ACS cannot be held responsible for errors or consequences arising from the use of information contained in these "Just Accepted" manuscripts. 


\title{
Viscous Force Retards Initial Droplet Spreading
}

\author{
Lichun Shi, 'Y Yang Liu, 'Hongyu Lu, ${ }^{\dagger}$ Yonggang Meng, ${ }^{\dagger}$ Guoqing Hu, ${ }^{\ddagger s}$ Yu Tian ${ }^{* \dagger}$ \\ ${ }^{\dagger}$ State Key Laboratory of Tribology, Tsinghua University, Beijing 100084, China
}

${ }^{\ddagger}$ State Key Laboratory of Nonlinear Mechanics, Institute of Mechanics, Chinese Academy of Sciences, Beijing 100190, China

${ }^{\S}$ School of Engineering Science, University of Chinese Academy of Sciences, Beijing 100049 , China.

\section{ABSTRACT}

A droplet could spread on a solid substrate within several milliseconds, and be divided into two stages, a fast inertial stage and a subsequent slow viscous stage. The effects of inertia and viscosity on spreading are presented with Ohnesorge number. A small Ohnesorge number corresponds to an initial inertial spreading. In this study, spreading experiments were carried out with a time resolution of $1 \mu \mathrm{s}$. Results disclose that the viscous force could retard droplet spreading at the beginning of liquid/solid surface contact, even with an Ohnesorge number smaller than 0.1 . According to simulation results, the viscous retardation could be ascribed to the real high local shear rate along the contact line at the spreading front. These results provide a new insight on droplet spreading dynamics and proposes, where exists a third viscous-inertial stage during the initial droplets spreading process. 


\section{INTRODUCTION}

Spreading of liquid droplets on a rigid flat surface, such as in raining $^{1}$, spin coatings ${ }^{2}$, painting $^{3}$, liquid adhesion ${ }^{4}$ and inkjet printing ${ }^{5,6}$, is universal and usually happens in a time scaled from milliseconds to seconds. During a spreading process, the droplet meniscus near the contact front is usually a circle. The surface energy differences among the liquid/solid, solid/air and liquid/air interfaces supply the driving force of liquid spreading. The inertial force and the viscous force act as resistance forces during spreading. Plenty studies have been carried out on the spreading dynamics of liquid due to its importance in science and engineering applications ${ }^{7-}$ 20.

During the process of liquid droplets spreading on a solid surface, an initial fast stage and a subsequent slow stage have been found ${ }^{7-10}$. The spreading radius $r(t)$ increases as $t^{1 / 2}$ in the fast stage $^{7}$ and as $t^{1 / 10}$ (known as Tanner's $1 \mathrm{law}^{8-10}$ ) in the slow stage, where $t$ denotes time. The duration time of fast stage is expressed as ${ }^{7}$

$$
\tau \sim\left(\frac{\rho \gamma R}{\eta^{2}}\right)^{1 / 8} \sqrt{\frac{\rho R^{3}}{\gamma}}
$$

where $\eta, \gamma, \rho, R$ are the viscosity, surface tension, density and radius of the pendant droplet, respectively. Equation (1) shows that the duration time of fast stage is mainly dependent on the droplet size. The ratio of inertial and viscous velocity, Ohnesorge number $O h=\eta / \sqrt{\gamma \rho R}$, indicates that a smaller $O h$ induces a longer time of fast stage. ${ }^{7}$

The fast stage is dominated by the inertia effect, whereas the slow stage is dominated by the viscosity of liquid. ${ }^{11,12}$ Researchers have experimentally investigated the effects of droplet size, viscosity of liquid and wettability of solid surface on spreading dynamics. ${ }^{7,13-16}$ The droplet size 
is also important in the fast stage, whereas the fluid viscosity is not. The spreading velocity increases as the droplet size increases. In the fast stage, the spreading is mainly resisted by the inertial force. ${ }^{7,13,14}$ The wettability of partial wetting solid surface has a minor effect on the initial fast spreading. However, the spreading velocity decreases with the increase of liquid/solid contact angle at later stage of fast spreading. ${ }^{13,15,16}$ During the slow spreading, the viscous friction hinders the liquid droplet spreading until the spreading radius reaches an equilibrium value. ${ }^{9,17}$ In this paper, the fast and slow stage are described as inertial stage and viscous stage, respectively.

In previous studies on the spreading dynamics, the geometrical evolution of liquid droplet was usually captured by a high-speed camera. ${ }^{18-20}$ However, it is very expensive to achieve a time resolution of $1 \mu \mathrm{s}$. Recently, an electrical method with a high temporal and spatial resolution has been proposed to characterize the spreading process of conductive droplets. Some new phenomena about two conductive droplets coalescence process have been disclosed. ${ }^{21-25}$ Case et al obtained the spreading velocity of bridge radius at $t<<10 \mu \mathrm{s}$, and proposed an earlier stage in which the two liquids coalesce with a slightly deformed cylindrical interface, and found the spreading velocity at $t<<10 \mu \mathrm{s}$ is contradictory to previous reports of viscous stage. ${ }^{21,22}$ Early $^{2}$ studies have identified the coalescence process of liquid drops has similar stages in liquid spreading. ${ }^{13,15,21}$ However, Paulsen et al captured the coalescence shapes of two silicone-oil drops by electrical measurements and found an earlier viscous-inertial stage ahead of the inertial stage and viscous stage. During the viscous-inertial stage, the viscous force, surface tension and inertial force are equally important in the coalescence process. ${ }^{23,24}$

However, such an important stage during droplets spreading has not been reported yet. The aim of this study is to characterize the effect of viscous force on the process of droplets 
spreading, especially on the beginning several milliseconds, with an electrical measurement and a finite element simulation. In this study, an equivalent circuit was proposed and selected a suitable frequency of AC power supply of the electrical method by analyzing the electrochemical impedance spectroscopy (EIS) of the liquid/solid interface system. By analyzing the highfrequency electrical signals, a relationship between the spreading radius and the resistance of liquid meniscus was deduced. By comparing the spreading velocity of liquids with same conductivity, the viscous force retarded droplet spreading was disclosed at the initial spreading time. In former researches, Eddi et al showed the variation of the spreading velocity for different viscosity droplets by recording images of pendent drops spreading on flat substrates. However, the spreading velocity only decreased slightly when the viscosity was larger than $220 \mathrm{mPa} \cdot \mathrm{s}$, corresponding to an Ohnesorge number larger than one. ${ }^{16}$ Moreover, to further understand the effect of viscous force on the dynamic wetting process, a finite element analysis using a level set method (COMSOL Multiphysics) was carried out to explain the phenomenon.

\section{EXPERIMENTAL SECTION}

2.1 Experimental apparatus. The conductive liquids used in the experiment were mixtures of potassium chloride $(\mathrm{KCl})$ solution and glycerin. Properties of these mixtures are listed in Table S1 of the Supporting Information (SI Section S1). The Oh of the liquids are much smaller than 0.1. Figure 1 shows the schematic of the experiment. Two parallel glass plates coated with a conductive Indium Tin Oxide (ITO) thin film (yellow layers) were used. A TopPette Pipettors (Dragon Lab) was used to manually put one droplet (with volume of $5 \mu \mathrm{L}$ ) on the lower ITO plate. The droplet radius $(R)$ on the glass plate is $\sim 1.7 \mathrm{~mm}$, which is smaller than the capillary 
length, ${ }^{26}$ therefore to neglect the gravity effect. Then the upper ITO glass plate was moved downward at a velocity of $\sim 3 \mu \mathrm{m} / \mathrm{s}$ to contact with the droplet. Once the upper plate contacted with the droplet, the motion was stopped.

The two ITO electrodes were connected in series with a resistor $\left(R_{0}=1000 \Omega\right)$ and a signal generator (Agilent Corporation) supplying sinusoidal AC power. The minimum voltage for water electrolysis to take place is up to $1.23 \mathrm{~V}$ at room temperature. ${ }^{27}$ To avoid electrolyzing, the amplitude of the AC power was set to $300 \mathrm{mV}$. During experiments, the voltage on $R_{0}$ and the AC power were recorded simultaneously with a PCI data acquisition (DAQ) card (Art Technology Development Corporation). The maximum acquisition rate is 80 million points per second. A high-speed camera (Optronis Camera) with a long-range working distance lens recorded the images of droplets at a rate of 1,922 frames/s.

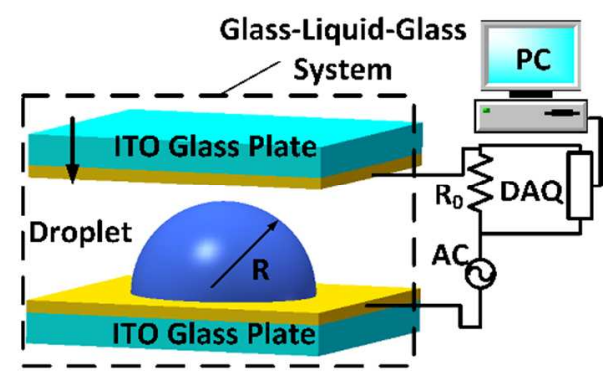

Figure 1. Schematic of the test system. The upper plate was moved down at a velocity $3 \mu \mathrm{m} / \mathrm{s}$. Once it touched the liquid drop, it was stopped.

2.2 Simulation. A two-dimensional axisymmetric model and a level set method were used to simulate the dynamic wetting process of droplet. More details on the level set method and the simulation model are presented in Section S2 of the SI. By considering the dynamic contact angle may vary with contact line velocity during droplet spreading, the correlation of dynamic contact angle and contact line velocity with the effect of viscosity of liquid is expressed as: ${ }^{28,29}$ 
$\cos \theta_{D}=\cos \theta_{0}-\frac{2 k_{B} T}{\gamma l^{2}} \operatorname{arcsinh}\left[\left(\frac{\eta V_{m}}{h}\right) \frac{v_{c l}}{2 \kappa_{s}^{0} l}\right] \quad$ (2),

where $k_{B}$ is the Boltzmann's constant, $T$ is the absolute temperature, $l$ is the average length of each molecular displacement, $h$ is the Planck's constant, $V_{m}$ is the molecular volume of water, $\kappa_{s}^{0}$ is the frequency of the molecular displacement and $v_{c l}$ is the velocity of contact line.

\section{RESULTS AND DISCUSSION}

3.1 Equivalent circuit. An electrochemical workstation (Autolab) was used to obtain the EIS of the glass-liquid-glass (GLG) system (Figure 1) after the droplet completely wetted the upper plate. Figure 2 shows the Bode (Figure 2a) and Nyquist (Figure 2b) plot of liquids with different viscosity and conductivity. $Z$ represents the total resistance of GLG system and Phase means the degree difference between electrical resistance $\left(Z^{\prime}\right)$ and capacitive impedance (- $Z$ ' '). According to Bard, the Bode and Nyquist plot proves that the GLG system consists of a resistor and a capacitor in series, because $\mathrm{Z}$ maintains a constant and $\mathrm{Z}$ ' becomes the dominant factor at high frequency. ${ }^{30}$ When the frequency is above $10^{4} \mathrm{~Hz}$, the phase angle is below 20 degrees and the electrical resistance keeps a stable value. It indicats that the frequency of AC power should be larger than about $10^{4} \mathrm{~Hz}$ to make the droplets electrical resistance be dominant during the dynamic spreading process. On this condition, we proposed a simplified circuit as shown in the inset of Figure $2 \mathrm{~b} . R_{\mathrm{S}}, \mathrm{C}_{\mathrm{p}}$ and $R_{\mathrm{ITO}}$ denote the droplet electrical resistance, the total capacitance of GLG system and the resistance of one ITO plate, respectively. The droplet electrical resistance is expressed as:

$$
R_{S}=\frac{U_{m} \cos \theta-U_{R_{0}}}{\frac{U_{R_{0}}}{R_{0}}}-2 R_{I T O}
$$


where $U_{m}$ is the amplitude of sine wave power, $U_{R_{0}}$ is the maximum voltage on $R_{0}$ in each cycle, and $\theta$ represents the phase difference between $U_{m}$ and $U_{R_{0}}$. When the AC power frequency is 1 $\mathrm{MHz}$, the time resolution could be up to $1 \mu \mathrm{s}$.
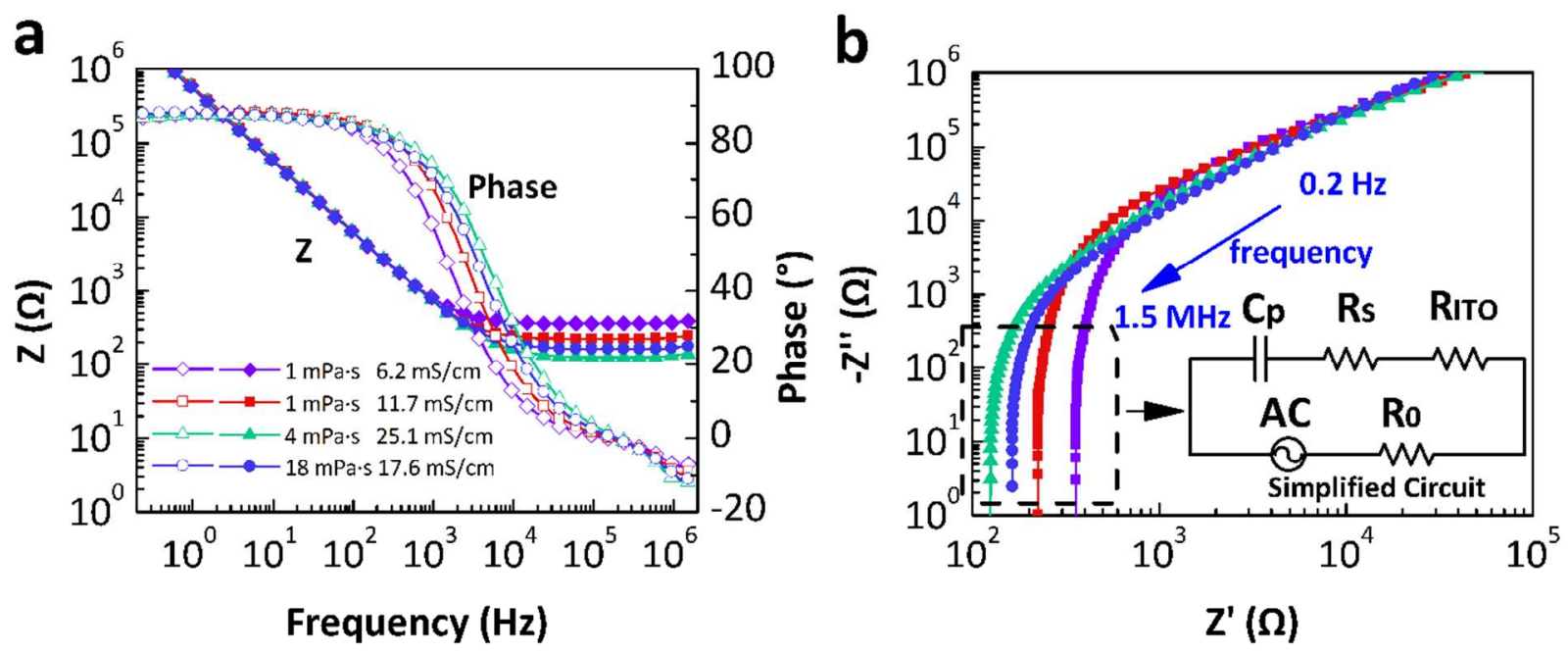

Figure 2. Bode (a) and Nyquist (b) plot of liquids with different viscosity and conductivity. Inset of (b) is a simplified circuit when the AC signal frequency is above $10^{4} \mathrm{~Hz}$.

\subsection{Relationship of spreading radius and droplets electrical resistance. Typical side and} top views of droplets during the first $4 \mathrm{~ms}$ of spreading are shown in Figure 3a. The spreading radius could be achieved from the top view images as shown in Figure $3 b$ (hollow symbols). The droplet began to deform at the top position and the capillary wave passed to the bottom rapidly as the spreading radius increasing. During the spreading process, the geometry of droplets turned from a sphere into a cone. The droplet electrical resistance, $R_{S}$, could be expressed as:

$R_{S}=\int_{0}^{h} \frac{1}{\sigma S} d y=\int_{0}^{h} \frac{1}{\sigma \pi(x(y))^{2}} d y$ 
where $h$ is the height of liquid droplet, $S$ and $x(y)$ are the section area and radius of dashed circle as Figure 3c shown. Equation (4) indicates that the electrical resistance is mainly dependent on the minimal section area. Figure $3 \mathrm{~d}$ shows the relationship between the droplet electrical resistance multiplied by conductivity $\left(\sigma R_{S}\right)$ and the reciprocal of the spreading radius $\left(1 / r_{\min }\right)$ obtained from images. Figure $3 \mathrm{~d}$ indicates that $\sigma R_{S}$ is linearly proportional to $1 / r_{\min }$, and $R_{S}$ could be described as

$R_{S}=\frac{\xi_{1}}{\sigma r_{\min }}-\frac{\xi_{2}}{\sigma R_{c o}}$

where $R_{c o}=1 \mathrm{~mm}$ is a fitting radius. The values of $\xi_{1}$ and $\xi_{2}$ of droplets with different conductivities are used to calculate the spreading radius as shown in Table S2 (SI Section S3).

Based on the above electrical method, the spreading radius was calculated by Equation (3) and (5). Comparison of spreading radius got by the electrical method and images is shown Figure $3 \mathrm{~b}$. The log-log inset of Figure $3 \mathrm{~b}$ shows the spreading radius $(1 \mathrm{mPa} \cdot \mathrm{s}, 11.7 \mathrm{mS} / \mathrm{cm})$ obtained from the electrical method agree well with that from the high speed camera (pure water spreading on a completely wetting surface) within $1 \mathrm{~ms}^{15,16}$ 

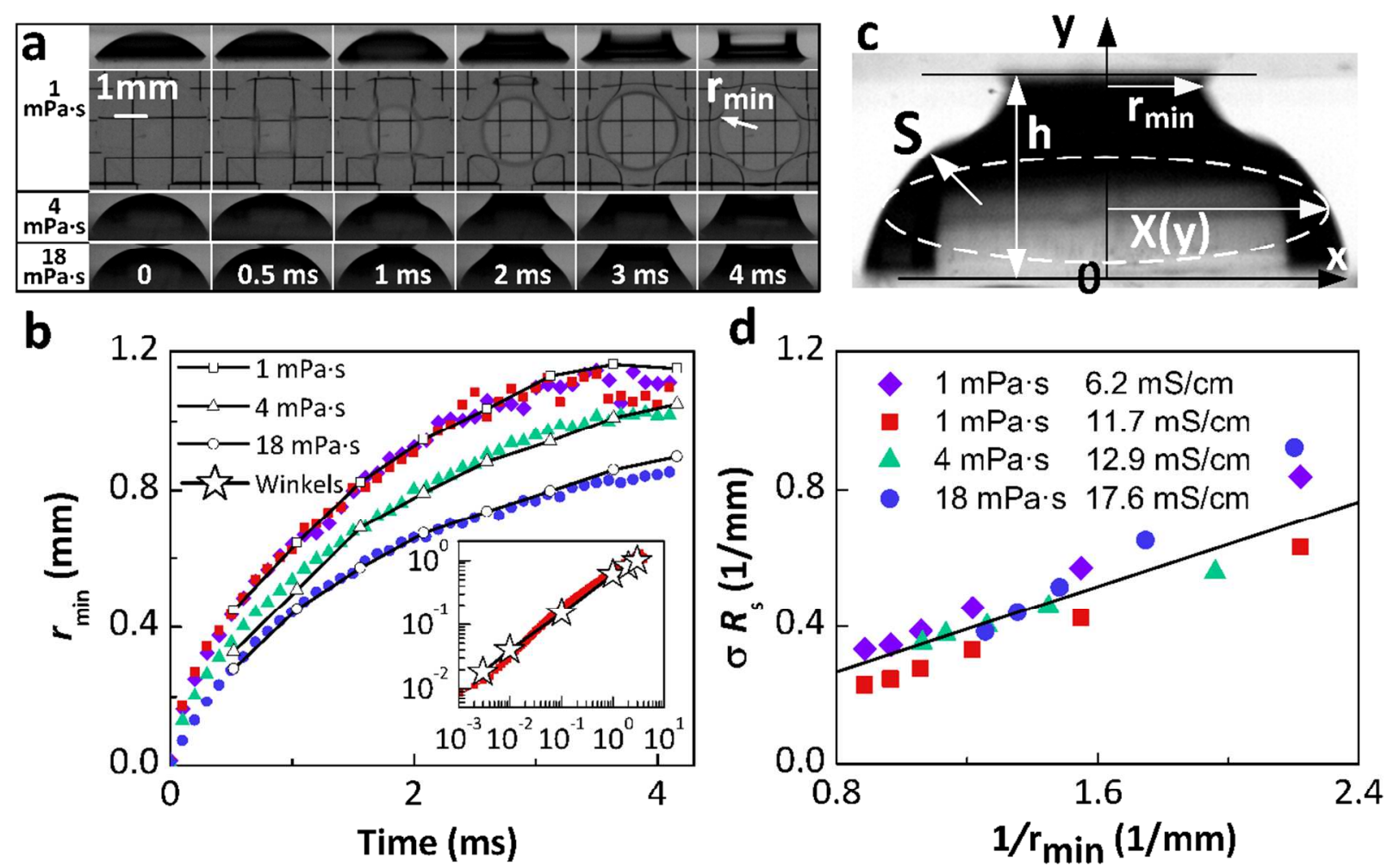

Figure 3. Typical experimental results. (a) Typical side view and top view of droplets with different viscosity during spreading. The white scale bar represents $1 \mathrm{~mm} . r_{\min }$ represents the spreading radius. (b) Evolution of spreading radius of liquid bridge as a function of time. The hollow symbols and solid ones represent data from pictures and calculated with resistance, respectively. (c) Geometry of one liquid droplet during spreading process. (d) Droplets electrical resistance multiplied by conductivity $\left(\sigma R_{S}\right)$ versus reciprocal of spreading radius $\left(1 / r_{\text {min }}\right)$.

\subsection{The effect of liquid conductivity on the electrical method. The spreading radius of} liquids with viscosity of $1 \mathrm{mPa} \cdot \mathrm{s}$ and different conductivity are shown in Figure 4 . When the conductivity is very low, the spreading radius tend to level off during the beginning $10 \mu \mathrm{s}$. The inset of Figure S3 (SI Section S3) proves that the electrical force does not affect the spreading 
radius at such low voltage. Through fitting the data in the Nyquist plot (Figure S4a in SI Section S4) obtained by the electrochemical workstation, the solution resistance $\left(R_{s l}\right)$ and the effective double-layer capacitance $\left(C_{d}\right)$ could be got as shown in Figure 5a. The effective double-layer capacitance can be expressed as ${ }^{31}$ :

$C_{d}=Q^{1 / n}\left(R_{s l}^{-1}+R_{p}^{-1}\right)^{(n-1) / n} \quad(6)$,

$Z_{C P E}=\frac{1}{Q(j 2 \pi f)^{n}}$

where $\mathrm{R}_{p}$ is the polarization resistance, $f$ represents the frequency of power supply. The value of the parameters in Equation (6) and (7) are shown in Table S3 (SI Section S4). The effective double-layer capacitance is almost the same for liquid with different conductivity and the solution resistance is inversely proportional to the liquid conductivity. The time constant $\left(\tau_{0}\right)$ for the $\mathrm{RC}$ circuit is expressed as:

$\tau_{0} \sim R_{s l} C_{d}$

The time constant is proportional to the solution resistance as shown in Figure 5a. When the liquid conductivity is larger than about $10 \mathrm{mS} / \mathrm{cm}$, the time constant will contain a very low value. 


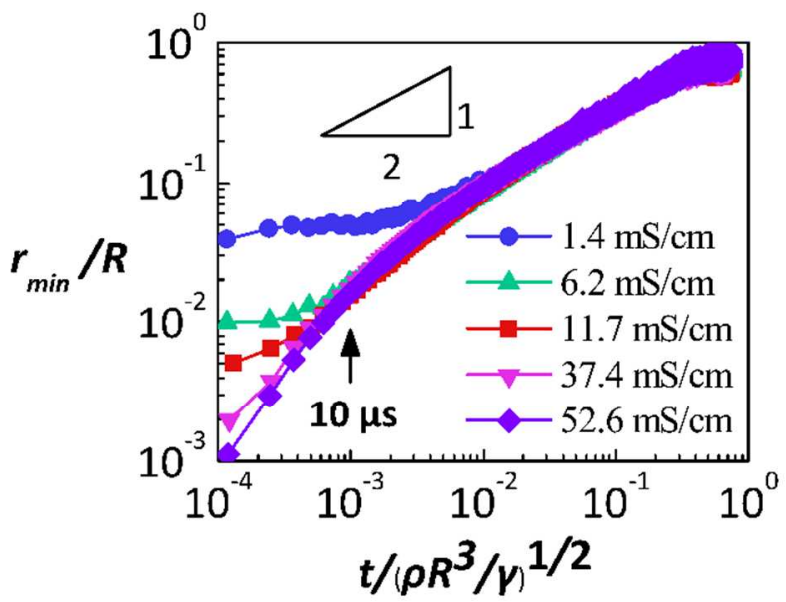

Figure 4. Spreading radius of liquids with a same viscosity of $\sim 1 \mathrm{mPa} \cdot \mathrm{s}$ and various conductivity at a range of $1.4-52.6 \mathrm{mS} / \mathrm{cm}$.

Circuit simulation was carried out by MATLLAB to reveal how the time constant affect the electrical method during droplets spreading process. The simulation circuit is shown in Figure S5 (SI Section S4). $R_{m}$, decreasing as $t^{\alpha_{m}}$, represents the droplet resistance during the wetting process. The inset of Figure $5 \mathrm{~b}$ shows the simulation time constant for liquids with different conductivity. The rapid discharge time of capacitance decreases with the decrease of the simulation time constant as shown in Figure $5 \mathrm{~b}$. The voltage on $R_{0}$ would not change with the law of spreading radius increasing during the capacitance rapid discharge time. That is the reason that when the conductivity is very low, the spreading radius tend to level off at early time as shown in Figure 4. As the spreading radius increase, the time constant will decrease and the spreading radius will be independent on liquid conductivity. Figure S4b (SI Section S4) shows that when conductivity is about $10-20 \mathrm{mS} / \mathrm{cm}$ the values of $C$ and $\alpha$ in Equation $\mathrm{S} 10$ (SI Section S3) are closed to 1.2 and 0.5 , which are in agreement with previous study results. ${ }^{15}$ 

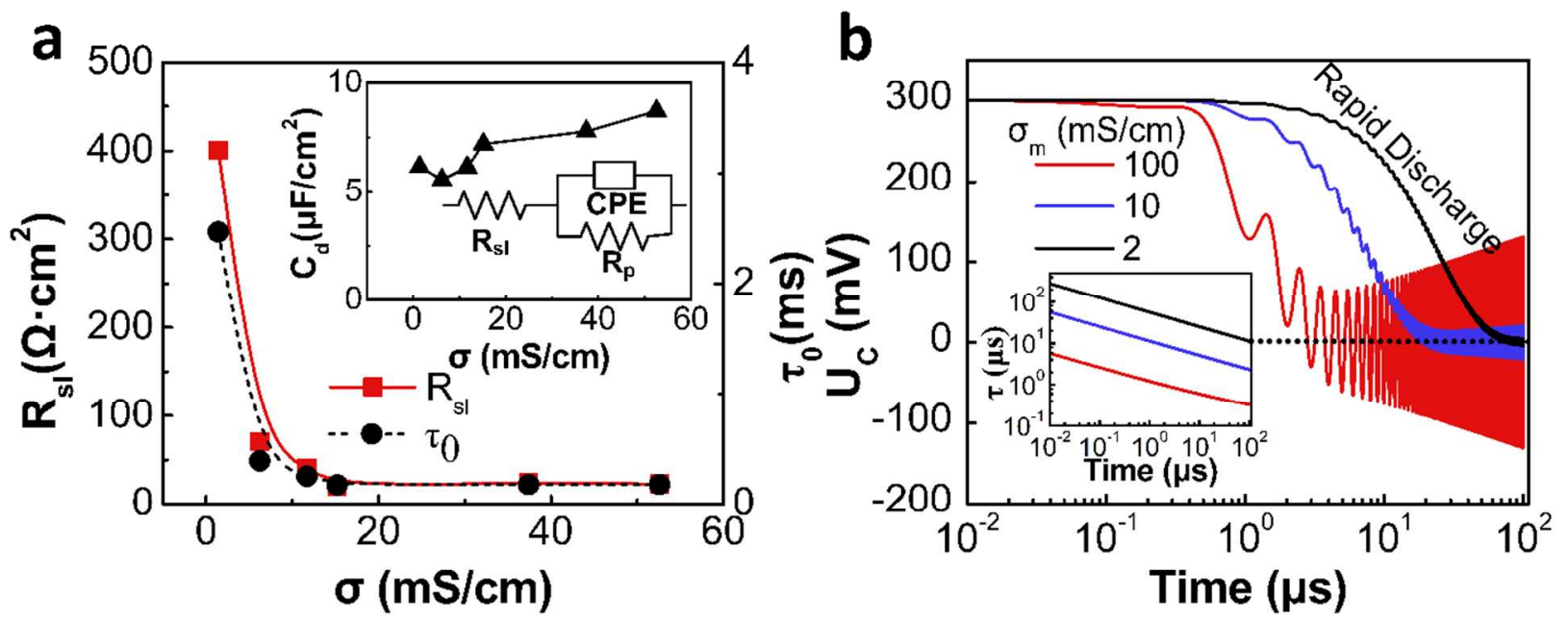

Figure 5. Simulation results. (a) The evolution of solution resistance $\left(R_{s l}\right)$ and time constant $\left(\tau_{0}\right)$ of $\mathrm{RC}$ circuit as a function of solution conductivity. The circuit in the inset is the equivalent circuits used to fit impedance data. (b) Simulation voltage of the capacitance varying with time.

3.4 The effect of liquids viscosity on the initial spreading. To understand the role of liquid viscosity on the initial spreading, spreading radius of liquids with conductivity in the range of $10-20 \mathrm{mS} / \mathrm{cm}$ and different viscosity are shown in Figure $6 \mathrm{a}$. The values of $C$ and $\alpha$ obtained from the data in Figure $6 \mathrm{a}$ within the initial $10 \mu \mathrm{s}$ are shown in Figure 6b. Figure $6 \mathrm{~b}$ indicates that the spreading velocity decreases significantly as the increase of liquid viscosity. It seems to be inconsistent with general description of that inertial force dominated initial spreading for $\mathrm{Oh}<1$. 

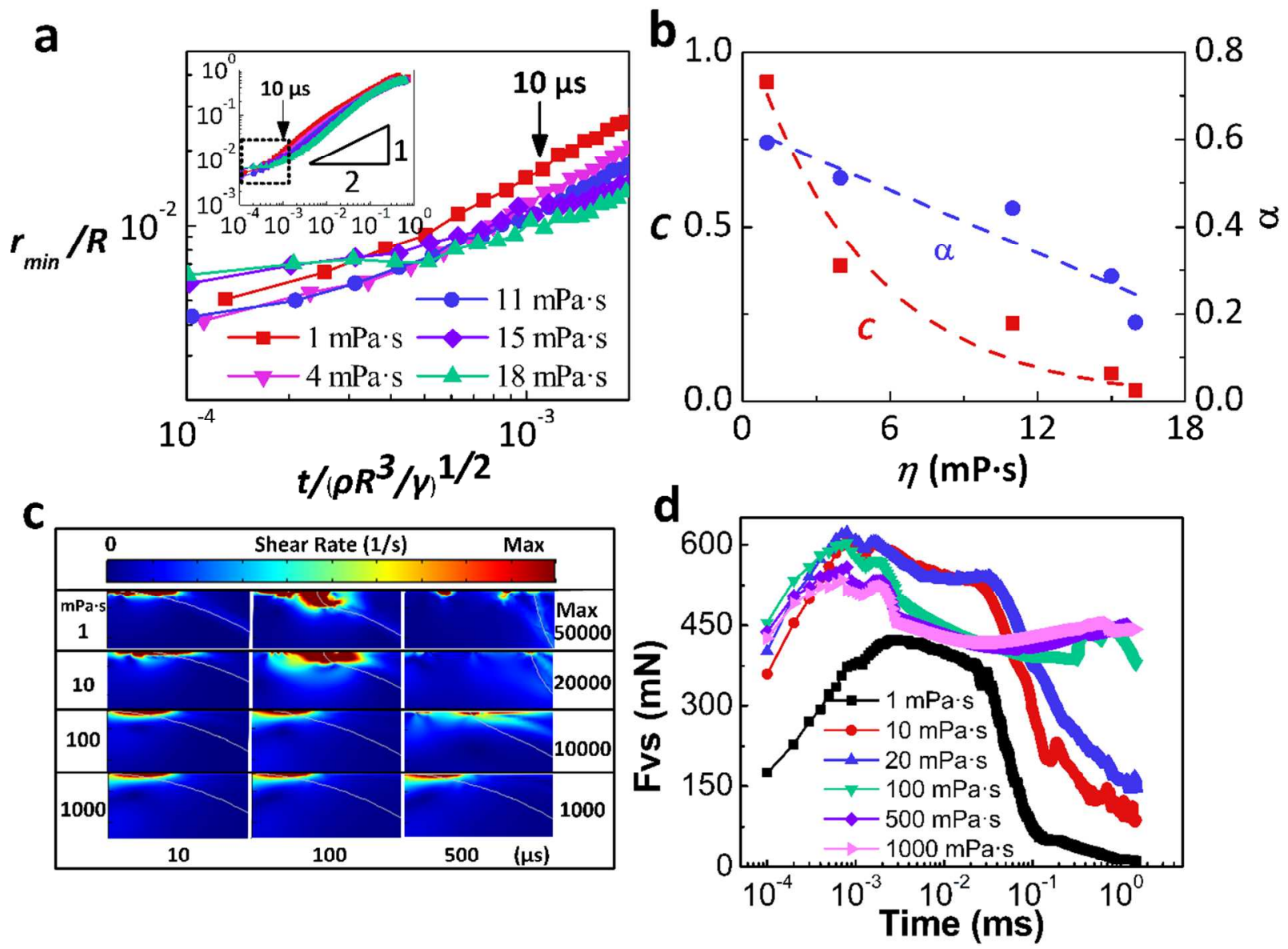

Figure 6. Comparison of spreading velocity and simulation viscous force for liquids with different viscosity. (a) Spreading radius of liquids with a same conductivity of $\sim 15 \mathrm{mS} / \mathrm{cm}$ and various viscosities. Figure (a) is the enlarged view of the dashed line area in the inset. (b) The value of $C$ and $\alpha$ versus viscosity. $C$ and $\alpha$ are obtained from the data in (a) during the first $10 \mu$ s. (c) Evolution of shear rate contour of different viscosity liquids as a function of spreading time. The results are the region encircled by the red dashed line in Figure S1a (SI Section S2). (d) Comparison of qualitative viscous force for liquids with different viscosity.

To explain the effect of viscosity on the initial spreading of droplets, simulation of the shear rate distribution of droplets at the spreading front was carried out. The simulation results showed 
that the viscous force is high at beginning of spreading even for low-viscosity liquids due to the high local shear rate at the spreading front.

Close-up images of the shear rate contour are shown in Figure 6c. The local shear rate along the contact line is much higher than other areas. For low-viscosity liquids $(1,10 \mathrm{mPa} \cdot \mathrm{s})$, the shear rate distributes on the upper plate decreases with time going. For high-viscosity liquids $(100,1000 \mathrm{mPa} \cdot \mathrm{s})$, the shear rate distribution does not change significantly as the spreading radius increase. The viscous force is defined as:

$F_{v s}=\eta \dot{\gamma}_{\text {ave }} L$

where $\dot{\gamma}_{\text {ave }}$ is the average shear rate distributed on the upper plate, $L$ is the length of the upper boundary in the simulation model. Using Equation (9), the viscous force during the dynamic wetting process was shown in Figure 6d. For low-viscosity liquids, the viscous force kept a high value during the initial $20 \mu \mathrm{s}$, and then decreased sharply. For high-viscosity liquids, the viscous force kept a high value during all the initial spreading. Base on the simulation, the viscous force could not be neglected during the initial spreading, even for low-viscosity liquids and macroscopic $\mathrm{Oh}<1$.

3.5 Viscous-inertial stage. Based on the results, we propose that there exists a viscous-inertial stage at the beginning of spreading before the inertial dominating stage as sketched in Figure 7. Once the distance between the surfaces of solid and liquid $(d)$ is smaller than about $10 \mathrm{~nm}$, the liquid surface jumps into contacting with the solid surface due to the attractive van der Waals force as shown in Figure $7 \mathrm{a}^{32,33}$ 
$F_{v d w}=-\frac{A_{s a l} R}{6 d^{2}}$

$(10)$,

where $A_{\text {sal }}$ is the Hamaker constant of the solid and liquid surfaces across air. The spreading radius varies from zero to several nanometers.

After the droplet contacts with the solid surface, the spreading radius increases rapidly form several nanometers to several micrometers as shown in Figure $7 \mathrm{~b}$. There is a meniscus on the liquid surface near the contact area. The curvature of the meniscus could be expressed as ${ }^{7,34}$

$r_{m} \sim \frac{R}{r^{2}}$

(11).

So the driven force $F_{L}$ caused by the Laplace pressure $\left(P_{L}\right)$ is ${ }^{7}$

$F_{L} \sim \pi r^{2} P_{L}=\pi r^{2} \gamma\left(\frac{1}{r}+\frac{1}{r_{m}}\right) \sim \pi \gamma r+\pi \gamma R$

The inertial force $F_{I}$, which is proportional to the mass of liquid $m$ entrained by the capillary force, is expressed as ${ }^{7}$

$F_{I} \sim m \sim \frac{\rho r^{4}}{R}$

When the spreading radius is very small, the inertial force could be ignored. The locally viscous force is

$F_{v}=\eta \dot{\gamma} \sim \eta \frac{d r}{r d t}$

where $\dot{\gamma}$ is the shear rate. The simulation results have showed that the real local shear rate distribution near the contact line at the spreading front is very large during the very beginning of spreading. Within the initial $10 \mu \mathrm{s}$, the dominant resistant force would be the viscous force, and 
the spreading velocity decreases with the increase of liquid viscosity as shown in Figure 6a and Figure $6 \mathrm{~b}$. After this period, the viscous force for low-viscosity liquid could decrease due to a lower shear rate near the contact line as shown in Figure 7c. The inertial force, which is proportional to $r^{4}$, starts to be considerably larger than the viscous force as sketched in Figure $7 \mathrm{c}$.
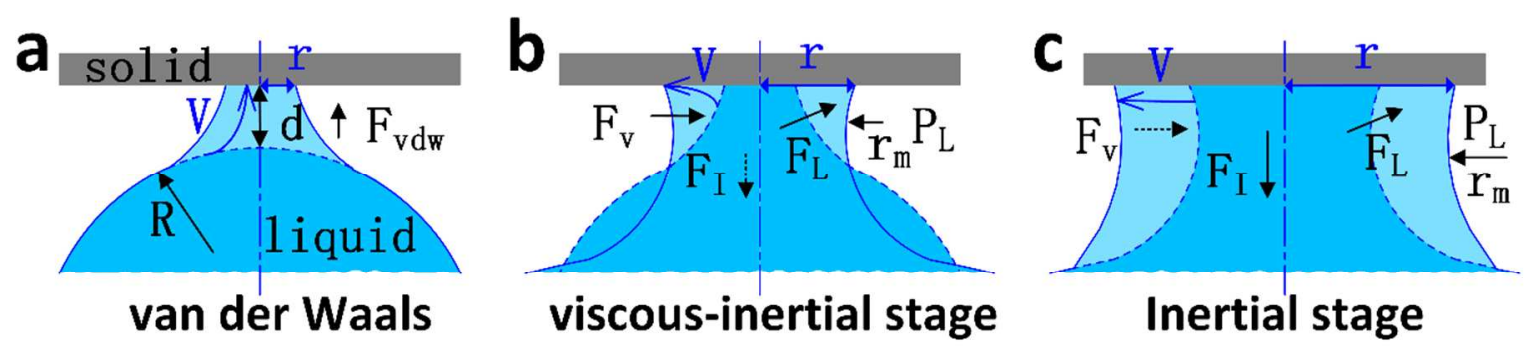

Figure 7. Schematic of three steps during droplets spreading. (a) The liquid surface jumps into the solid surface due to the attractive van der Waals force with a spreading radius varies from zero to $r$. (b) After initial contact, the spreading radius grows rapidly with a high local shear rate near the contact line. (c) The inertial force increases with spreading radius and becomes dominant.

\section{CONCLUSIONS}

In summary, an electrical method was used to reveal the role of viscous force on the process of liquid droplets spreading on a solid substrate. Spreading dynamics of a conductive droplet on an upper plate was investigated by measuring its electrical resistance with a time resolution of $1 \mu \mathrm{s}$. This fast electrical method discloses that the spreading velocity decreases as liquids viscosity increases at the beginning spreading even for low-viscosity liquids, which indicates a viscousinertial stage might exist before inertial stage during droplets spreading. 
A finite element simulation using a level set method was carried out to obtain more dynamic flow details The simulation results show that the local shear rate near the contact line is much higher than other regions, particularly at the beginning of spreading. This is the reason that the viscous force is the major resistant force during the initial spreading time. This study provides a new insight on the transient behaviors of liquid spreading.

\title{
ASSOCIATED CONTENT
}

\section{Supporting Information}

Properties of liquids; Simulation method; Comparison of spreading radius got by electrical measurement and images, The effect of liquids conductivity on the electrical method (PDF)

\section{AUTHOR INFORMATION}

\section{Corresponding Author}

*E-mail: tianyu@tsinghua.edu.cn. Tel: +86-10-62782981.

\section{Author Contributions}

The manuscript was written through contributions of all authors. All authors have given approval to the final version of the manuscript.

\author{
Notes \\ The authors declare no competing financial interest.

\section{ACKNOWLEDGMENTS} \\ This work is sponsored by the National Natural Science Foundation of China (Grant No. \\ 51425502 and 51323006).
}




\section{REFERENCES}

(1) Bergeron, V.; Bonn, D.; Martin, J. Y.; Vovelle, L. Controlling Droplet Deposition with Polymer Additives. Nature 2000, 405, 772-775.

(2) Simpkins, P. G.; Kuck, V. J. On Air Entrainment in Coatings. J. Colloid Interface Sci. 2003, 263, 562-571.

(3) Hwang, J. K.; Cho, S.; Dang, J. M.; Kwak, E. B.; Song, K.; Moon, J.; Sung, M. M. Direct Nanoprinting by Liquid-Bridge-Mediated Nanotransfer Moulding. Nat. Nanotechnol. 2010, $5,742-748$.

(4) Grilli, S.; Coppola, S.; Vespini, V.; Merola, F.; Finizio, A.; Ferraro, P. 3D Lithography by Rapid Curing of the Liquid Instabilities at Nanoscale. Proc. Natl. Acad. Sci. U. S. A. 2011, $108,15106-15111$

(5) Toda, M.; Chen, Y.; Nett, S. K.; Itakura, A. N.; Gutmann, J.; Berger, R. Thin Polyelectrolyte Multilayers Made by Inkjet Printing and their Characterization by Nanomechanical Cantilever Sensors. J. Phys. Chem. C 2014, 118, 8071-8078.

(6) Sykora, B.; Wang, D.; von Seggern, H. Multiple Ink-Jet Printed Zinc Tin Oxide Layers with Improved TFT Performance. Appl. Phys. Lett. 2016, 109, 033501.

(7) Biance, A. L.; Clanet, C.; Quéré, D. First Steps in the Spreading of a Liquid Droplet. Phys. Rev. E 2004, 69, 016301.

(8) Bonn, D.; Eggers, J.; Indekeu, J.; Meunier, J.; Rolley, E. Wetting and Spreading. Rev. Mod. Phys. 2009, 81, 739.

(9) Rafaï, S.; Sarker, D.; Bergeron, V.; Meunier, J.; Bonn, D. Superspreading: Aqueous Surfactant Drops Spreading on Hydrophobic Surfaces. Langmuir 2002, 18, 10486-10488. 
(10) Tanner, L. H. The Spreading of Silicone Oil Drops on Horizontal Surfaces. J. Phys. D: Appl. Phys. 1979, 12, 1473.

(11) Chen, L. Bonaccurso, E. Electrowetting-from Statics to Dynamics. Adv. Colloid Interface Sci. 2014, 210, 2-12.

(12) Ralston, J.; Popescu, M.; Sedev, R. Dynamics of Wetting from an Experimental Point of View. Annu. Rev. Mater. Res. 2008, 38, 23-43.

(13) Bird, J. C.; Mandre, S.; Stone, H. A. Short-Time Dynamics of Partial Wetting. Phys. Rev. Lett. 2008, 100, 234501.

(14) Courbin, L.; Bird, J. C.; Reyssat, M.; Stone, H. A. Dynamics of Wetting: from Inertial Spreading to Viscous Imbibition. J. Phys.: Condens. Matter 2009, 21, 464127.

(15) Winkels, K. G.; Weijs, J. H.; Eddi, A.; Snoeijer, J. H. Initial Spreading of Low-Viscosity Drops on Partially Wetting Surfaces. Phys. Rev. E 2012, 85, 055301.

(16) Eddi, A.; Winkels, K. G.; Snoeijer, J. H. Short Time Dynamics of Viscous Drop Spreading. Phys. Fluids 2013, 25, 013102.

(17) De Gennes, P. G. Wetting: Statics and Dynamics. Rev. Mod. Phys. 1985, 57, 827.

(18) Carlson, A.; Bellani, G.; Amberg, G. Contact Line Dissipation in Short-Time Dynamic Wetting. Europhys. Lett. 2012, 97, 44004.

(19) Lee, J. B.; Lee, S. H. Dynamic Wetting and Spreading Characteristics of a Liquid Droplet Impinging on Hydrophobic Textured Surfaces. Langmuir 2011, 27, 6565-6573.

(20) Yang, C.; Burrous, A.; Xie, J.; Shaikh, H.; Elike-Avion, A.; Rojas Rodriguez, L.; Ramachandran, A.; Choi, W.; Mazzeo, A. D. High-Gravity Spreading of Liquid Puddles on Wetting Flexible Substrates. Appl. Phys. Lett. 2016, 108, 074102. 
(21) Case, S. C.; Nagel, S. R. Coalescence in Low-Viscosity Liquids. Phys. Rev. Lett. 2008, 100, 084503.

(22) Case, S. C. Coalescence of Low-Viscosity Fluids in Air. Phys. Rev. E 2009, 79, 026307.

(23) Paulsen, J. D.; Burton, J. C.; Nagel, S. R. Viscous to Inertial Crossover in Liquid Drop Coalescence. Phys. Rev. Lett. 2011, 106, 114501.

(24) Paulsen, J. D.; Burton, J. C.; Nagel, S. R.; Appathurai, S.; Harris, M. T.; Basaran, O. A. The Inexorable Resistance of Inertia Determines the Initial Regime of Drop Coalescence. Proc. Natl. Acad. Sci. U. S. A. 2012, 109, 6857-6861.

(25) Paulsen, J. D. Approach and Coalescence of Liquid Drops in Air. Phys. Rev. E 2013, 88, 063010.

(26) De Gennes, P. G.; Brochard-Wyart, F.; Quéré, D. Capillarity and Wetting Phenomena: Drops, Bubbles, Pearls, Waves; Springer: New York, 2013.

(27) Ursua, A.; Gandia, L. M.; Sanchis, P. Hydrogen Production from Water Electrolysis: Current Status and Future Trends. Proc. IEEE 2012, 100, 410-426.

(28) Blake, T. D.; De Coninck, J. The Influence of Solid-Liquid Interactions on Dynamic Wetting. Adv. Colloid Interface Sci. 2002, 96, 21-36.

(29) Chen, J.; Zhang, Z.; Xu, R.; Ouyang, X.; Jiang, P. Numerical Investigation of the Flow Dynamics and Evaporative Cooling of Water Droplets Impinging onto Heated Surfaces: An Effective Approach to Identify Spray Cooling Mechanisms. Langmuir 2016, 32, 91359155.

(30) Bard, A. J.; Faulkner, L. R. Electrochemical Methods: Fundamentals and Applications; Wiley: New York, 1980. 
(31) Harrington S P, Devine T M. Relation between the Semiconducting Properties of a Passive Film and Reduction Reaction Rates. J. Electrochem. Soc., 2009, 156, C154-C159.

(32) Zeng, H.; Tian, Y.; Zhao, B.; Tirrell, M.; Israelachvili, J. Transient Interfacial Patterns and Instabilities Associated with Liquid Film Adhesion and Spreading. Langmuir 2007, 23, 6126-6135.

(33) Zeng, H.; Zhao, B.; Tian, Y.; Tirrell, M.; Leal, L. G.; Israelachvili, J. N. Transient Surface Patterns during Adhesion and Coalescence of Thin Liquid Films. Soft Matter 2007, 3, 8893.

(34) Eggers, J.; Lister, J. R.; Stone, H. A. Coalescence of Liquid Drops. J. Fluid Mech. 1999, 401, 293-310. 


\section{TOC Graphic}

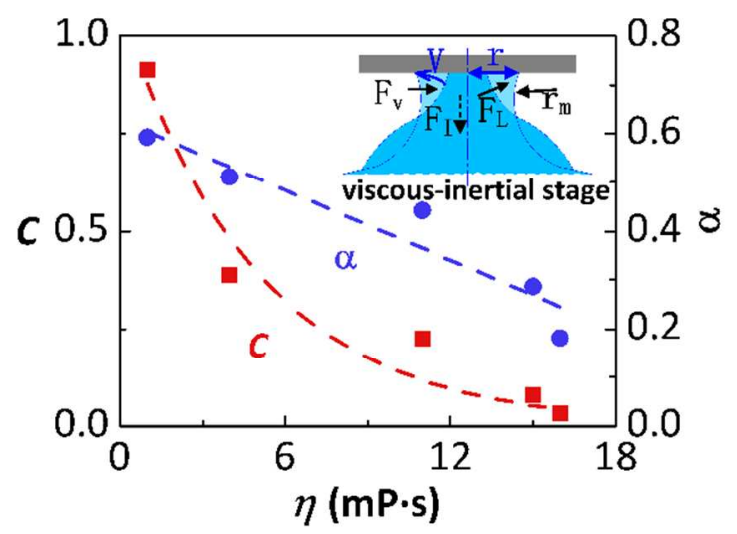

20

21 